\title{
BJMHR
}

British Journal of Medical and Health Research

Journal home page: www.bjmhr.com

\section{Importance of Ultrasonographic guided aspirated sample in early diagnosis of Musculoskeletal tuberculosis among the patients attending tertiary health care center.}

\author{
Vikramjeet Singh', Anil Chandra Phukan², Bhaskar Borgohain ${ }^{3}$ \\ 1.Department of Microbiology, Dr. Ram Manohar Lohia Institute of Medical Sciences, \\ Lucknow \\ 2.Department of Microbiology, North Eastern Indira Gandhi Regional Institute of Health and \\ Medical Sciences, Shillong. \\ 3.Department of Orthopaedics and Trauma, North Eastern Indira Gandhi Regional Institute \\ of Health and Medical Sciences, Shillong.
}

\section{ABSTRACT}

Clinicians consider musculoskeletal tuberculosis as diagnostic dilemma because diagnosis frequently gets delayed due to its varied clinical presentation, false negative results on smear microscopy and less sensitivity on culture methods (20-40\%). The main objective was to know the effectiveness of using ultrasonographic guided aspirate sample in early diagnosis of musculoskeletal tuberculosis. In this study, a total of thirty seven clinical specimens like pus swab and ultrasonography guided (USG) pus aspiration of newly suspected musculoskeletal tuberculosis patients were evaluated for microscopy, culture and molecular detection of $M$. tuberculosis. The study reveals prevalence of $45.9 \%$ musculoskeletal tuberculosis among the clinically suspected patients. The most commonly involved sites in suspected musculoskeletal tuberculosis were hip joint (23.1\%). USG guided pus comprises only $26.9 \%$ of the samples collected, however, yield of $M$. tuberculosis from them were $71 \%$. PCR detected the maximum number of cases of musculoskeletal tuberculosis 17 (45.9\%) followed by culture method $9(24 \%)$ and smear microscopy $1(2.7 \%)$. This observation will help guiding clinicians in effective management of musculoskeletal tuberculosis cases because delayed diagnosis and treatment in musculoskeletal TB results in poor outcome.

Keywords: Polymerase Chain reaction, Extrapulmonary tuberculosis, Osteoarticular, USG 


\section{INTRODUCTION}

In Extrapulmonary TB (EPTB) highly vascular areas such as lymph nodes, pleura, genitourinary tract, bones and joints and meninges are commonly affected. Although musculoskeletal TB comprises $10-15 \%$ of all extrapulmonary TB, it is difficult to diagnose with conventional bacteriological methods like acid fast staining, fluorescent microscopy and culture methods due to lack of representative samples ${ }^{1,2}$. The diagnostic dilemma and the challenges are many: firstly, skeletal TB is a paucibacillary condition; secondly it mimics other common musculoskeletal conditions like bone tumors, rheumatoid arthritis, osteoporotic vertebral fracture, and other infective conditions. Finally, paucity of proper microbiological settings and lack of specialized persons for collection and handling of laboratory samples needs urgent attention as there is paucity of studies to the best of our knowledge on musculoskeletal tuberculosis. Considering the fact mentioned above the present study has been undertaken with the intent to study the prevalence of musculoskeletal tuberculosis among the patients attending tertiary health care centre and to provide a rapid and reproducible methodology for making definitive diagnosis of musculoskeletal tuberculosis using samples, obtained in a less invasive manner like USG guided aspiration whenever feasible, from clinically suspected musculoskeletal tuberculosis patients.

\section{MATERIALS AND METHOD}

A total of thirty seven clinically suspected musculoskeletal tuberculosis patients fulfilling the inclusion criteria based on sign and symptoms like pain, restricted joint movements, swelling of the joints and other constitutional symptoms were included in this study. The study was conducted after obtaining informed consent of the participant on approval of the ethical clearance of the institute. The collected specimens like pus swab and USG guided pus aspirate, were processed and subjected for smear microscopy for demonstration of Acid Fast Bacilli (AFB), isolation of M. tuberculosis through in-vitro culture and molecular analysis employing PCR assay for confirmation of laboratory diagnosis using laboratory diagnostic techniques described elsewhere. ${ }^{3}$

PCR for TB was done using a specific sequence IS6110 (390 base pairs) primer. PCR was carried out employing utmost precautions against cross- and carryover contamination. Genomic DNA was extracted from various specimens using DNA-sorb-B nucleic acid extraction kit (REF K1-2-100-CE, AMPLISENS, Moscow, Russia) as per manufacturer's protocol. Mycobacterium tuberculosis DNA was amplified from $10 \mu \mathrm{l}$ of extracted sample using AmpliSensMBT-EPh PCR kit (REF B15-100-R0, 5-CE, AMPLISENS, Moscow, Russia) as per Manufacturer's Protocol. DNA amplification of 390bp IS6110 Insertion element was carried by two oligonucleotide primer IS6110f ( 5'- GGC AAA GCA GCT CTC 
TCT GC-3') and IS6110r (5'- GGA CTG CCA CCT TCC ATC TTC-3') in thermocycler DNA PCR machine (CG Palm Cycler 9600, Genetix Biotech, New Delhi) which were incorporated in PCR tubes. Amplified DNA was electrophoresed using 1.7\% agarose gel at 90 Volts for $1 \mathrm{hr}$ and the resultant bands were interpreted by UV transillumination (ethidium bromide).

\section{RESULTS AND DISCUSSION}

The study shows the prevalence of $45.9 \%$ musculoskeletal tuberculosis among the clinically suspected patients of musculoskeletal tuberculosis and indicated slight preponderance of females $(54.2 \%)$ in musculoskeletal tuberculosis than males (45.8\%). Majority of the patients affected by musculoskeletal tuberculosis were in the age group of 21-30 years (28.9\%) followed by pediatric age group (17.3\%).

The most commonly involved sites in suspected musculoskeletal tuberculosis were hip joint (23.1\%) and knee joint (23.1\%) followed by lumbar spine (19.2\%) and long bones of lower limb (17.4\%). Majority cases of musculoskeletal tuberculosis involved Hip joint 10 cases (41.67\%), with equal involvement of left and right side. Knee joint was involved in $25 \%$ and lumbar spine in $12.5 \%$ cases of musculoskeletal tuberculosis. Long bones of lower limb like tibia and femur were involved in $16.6 \%$ cases of musculoskeletal tuberculosis.

Among 37 clinical samples of suspected musculoskeletal tuberculosis patients, pus swabs 23 (62\%) were collected from joints like hip joint, knee joint, ankle joint and discharge from bones like tibia, femur, spine, upper limb bones etc, followed by ultrasonography guided pus aspiration (38\%). USG guided pus comprise only $38 \%$ of the sample collected however, yield of $M$. tuberculosis from them were $71 \%$, making them better samples in comparison to pus swab which had a poor yield of $30.4 \%$.

All 37 clinical specimens from newly suspected musculoskeletal tuberculosis were subjected for conventional and fluorescent microscopy (Image 1 and 2), however only 1 USG guided pus aspirate from hip joint demonstrated AFB (2.7\%). There was no significant difference between the detection of tubercle bacilli by conventional and fluorescent microscopy. Among all samples subjected for in-vitro culture on LJ slant, 9 clinical specimens (24\%) demonstrated growth of colonies. USG guided pus aspirate from hip joint (46.2\%) reported maximum growth on LJ slant.

In the present study, PCR detected the maximum number of cases of musculoskeletal tuberculosis $17(45.9 \%)$ followed by culture method $9(24 \%)$ and smear microscopy 1 $(2.7 \%)$.

Osteoarticular tuberculosis can cause significant morbidity and a high index of suspicion is needed for early diagnosis so as to avoid bone destruction and disability. ${ }^{2}$ In the study, female 
preponderance $(54.2 \%)$ was seen in musculoskeletal tuberculosis compared to males $(45.8 \%)$. Such observation did not reveal any significant difference of disease in male or female. Our observations were in concordance with that of Jutte et $\mathrm{al}^{\mathbf{6}}$ and Yoon et $\mathrm{al}^{4}$. However, compared to our study Nasiri et $\mathrm{al}^{7}$ and Enache et $\mathrm{al}^{\mathbf{8}}$, described higher preponderance among the males.

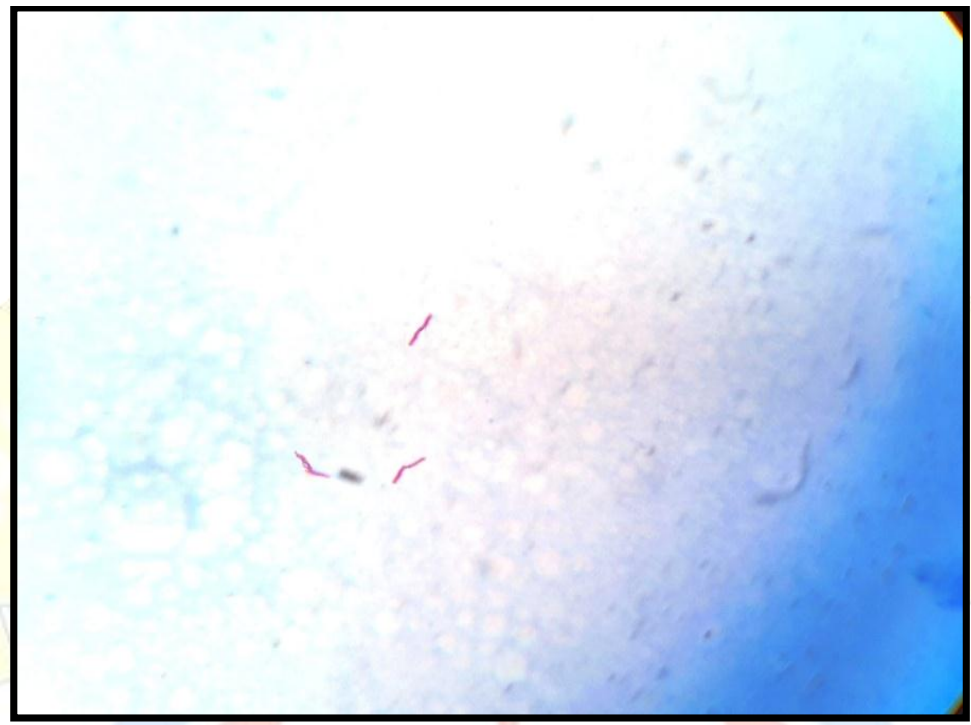

Image 1: Conventional Microscopy showing scattered Acid Fast Bacilli on Ziehl Neelsen Staining using USG aspirate sample

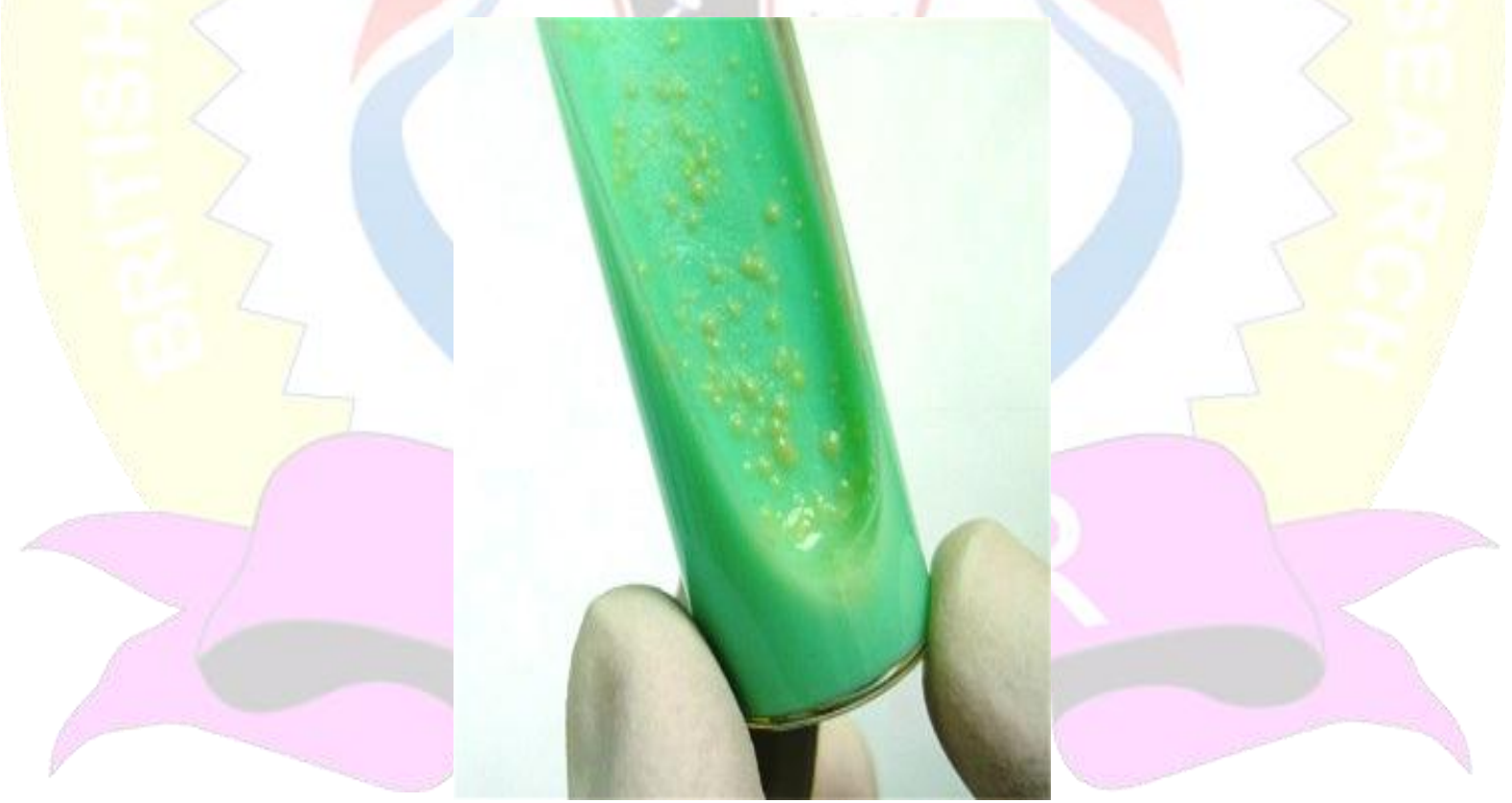

Image 2 : Lowenstein Jensen culture medium showing growth of Mycobacterium tuberculosis colonies from 


\section{PCNC434448495051 52}

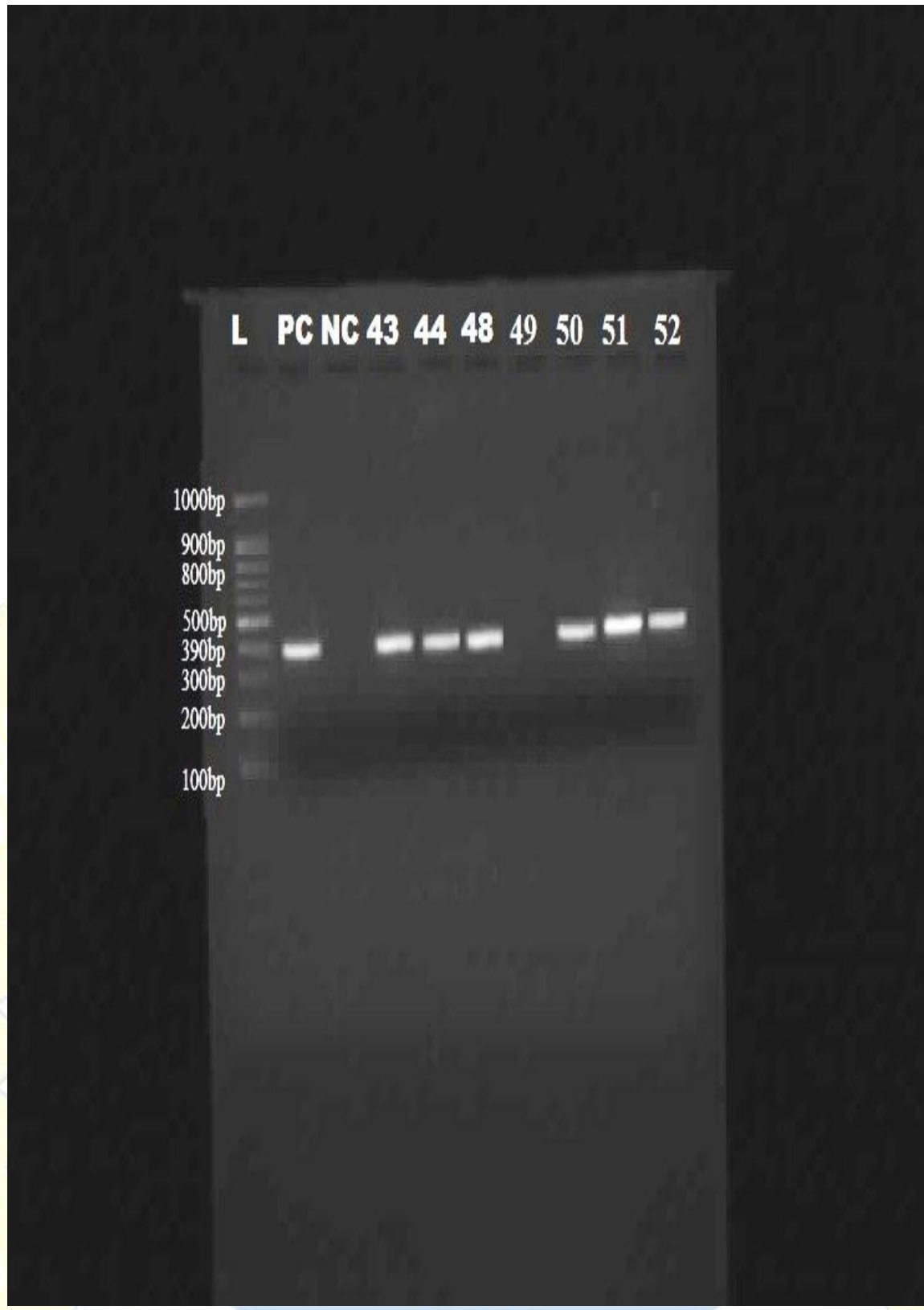

Image 3 : PCR amplification of 390bp in IS6110 region of $M . t b$ on $1.7 \%$ agarose gel [L: DNA ladder 1000bp to 100bp; PC: Positive Control; NC: Negative control] Lane 49: Negative, Lane 43, 44, 48, 50, 51, 52: Positive

Among 37 cases of clinically suspected musculoskeletal tuberculosis, the most commonly affected age group was between $21-30$ years (42\%) followed by pediatric age group $(20.2 \%)$. This observation was similar to the findings of Arathi et al ${ }^{\mathbf{9}}(37 \%)$ and Sharma et al ${ }^{10}(40 \%)$. However, there was difference in the observation with the study of Enache et $\mathrm{al}^{\mathbf{8}}(21 \%)$ where the mean age group was more than 50years.

In our study highest numbers of the cases were represented by suspected patient with joint tuberculosis cases $(55.8 \%)$ where hip joint 12 cases $(23 \%)$, knee joint 12 cases $(23 \%)$ and ankle joint 5 cases $(9.6 \%)$. The study indicated highest detection 10 cases $(41.67 \%)$ of tubercular lesions affecting the hip joints with equal involvement of both right and left side. 
The involvement of hip joint in present study was in accordance with the study reported by Sharma et $\mathrm{al}^{\mathbf{1 0}}(27 \%)$ and Enache et $\mathrm{al}^{\mathbf{8}}$ (31\%). The findings for Hip joint tuberculosis were inconsistent with the studies of Ruiz et $a l^{\mathbf{1 1}}(15.4 \%)$ and Sandher et $\mathrm{al}^{\mathbf{1 2}}$ (2.38\%). In our study 10 cases (19.2\%) were suspected of lumbar spine tuberculosis among 52 cases of musculoskeletal tuberculosis. The study reported 3 cases $(12.5 \%)$ with tubercular lesion affecting lumbar spine, this observation was similar to the study of Ruiz et al ${ }^{\mathbf{1 1}}(15.4 \%)$. However, these observations varies from the study of Sharma et $\mathrm{al}^{\mathbf{1 0}}(50.77 \%)$, Sandher et $\mathrm{al}^{\mathbf{1 2}}(44 \%)$. The reason for this variation could be difference in geographic and genetic variability of the study population.

In our study we found that $90.4 \%$ patients presented with pain and difficulty while walking, followed by backache in $55.8 \%$ patients and discharge from joints in 25 patients $(48.1 \%)$. These findings compare favorably with those of Arathi et $\mathrm{al}^{\mathbf{9}}$ and Ruiz et al ${ }^{\mathbf{1 1}}$ where pain $(83 \%)$, was the presenting complaint in majority of the patients. Constitutional symptoms of tuberculosis like fever and weight loss were reported in $24(46.2 \%)$ and 22 patients $(42.3 \%)$ respectively which was consistent with the study of Sharma et $\mathrm{al}^{\mathbf{1 0}}(45 \%)$, Sandher et $\mathrm{al}^{\mathbf{1 2}}$ $(39 \%)$.

In the present study, as shown in Table 1 total of 37 specimens received, majority of the sample collected were pus swabs $(62 \%)$, followed by ultrasonography guided pus aspiration (38\%). This observation was in corroboration with the findings of Prakash et al., 2016 ${ }^{\mathbf{1 3}}$ (31.6\%) and Kumar et al., 2014 ${ }^{\mathbf{1 5}}$ (56.4\%). Among all the 14 USG guided pus samples collected, M.tuberculosis was detected in 71\% (10 out of 14), showing significant yield in comparison to pus swab which had a poor yield of $30.4 \%$ (7 positive out of 23 ). Similar observation regarding poor yield of pus was also reported in the study at Karnataka by Ganavalli et al., $2013^{14}$ (25.8\%) and at two tertiary centres in Lucknow by Maurya et al., $2016^{\mathbf{2 1}}(27.65 \%)$, However, these findings differ from the study of Prakash et al., $2016^{\mathbf{1 3}}$ (43.24\%) and Ruiz et al., $2003^{11}(66.67 \%)$. Sample taken through USG guided aspiration showed better yield in cases of musculoskeletal tuberculosis.

In this study we have attempted to investigate the relevance of PCR assay in clinically suspected cases of musculoskeletal tuberculosis using pus swab and USG guided aspirate. In the present study (Table 2) the high degree of detection was apparent in the actual clinical setting, since PCR detected 17 among 37 cases studied, of which culture was positive in only 9 cases $(24.9 \%)$ and smear microscopy detected AFB in one case $(2.7 \%)$. The findings of PCR in this study compare favourably with Muangchan et $\mathrm{al}^{\mathbf{1 8}}(33.3 \%)$ and Prakash $\mathbf{J}$ et $\mathrm{al}^{\mathbf{1 3}}$ (40.1\%). However, differs from the study of Pandey $\mathrm{V}$ et $\mathrm{al}^{\mathbf{1 9}}(70.83 \%)$ and Negi SS et $\mathrm{al}^{\mathbf{2 0}}$ (78.2\%). Among the 23 pus swab samples, only 3 (13\%) samples showed growth of $M$. 
tuberculosis, smear microscopy did not detected any AFB from pus swab, however, PCR assay reported 7 pus swab samples (30.4\%) positive for M.tuberculosis. Among the 20 pus swab specimens which were negative by conventional staining and culture methods, PCR detected 4 positive cases $(20 \%)$ of musculoskeletal tuberculosis. The findings related to poor yield of pus was also reported in the study conducted by Ganavalli et al ${ }^{14}(25.8 \%)$ and Maurya et $\mathrm{al}^{21}(27.65 \%)$, However, these findings were inconsistent from the study of Prakash et $\mathrm{al}^{\mathbf{1 3}}(43.24 \%)$ and Ruiz et $\mathrm{al}^{\mathbf{1 1}}(66.67 \%)$. In the study 14 USG guided pus aspirate samples were collected from suspected musculoskeletal tuberculosis patients, among them 1 (7\%) was positive on smear microscopy and $6(42.9 \%)$ were positive on LJ culture medium, however, PCR demonstrated 10 positive (71.4\%) samples. Among the 8 USG guided pus aspirate samples which were not demonstrated on conventional and fluorescent microscopy and culture, PCR reported 4 positive samples (50\%).

Table 1: Distributions of the clinical specimens collected from different sites with suspected musculoskeletal tuberculosis

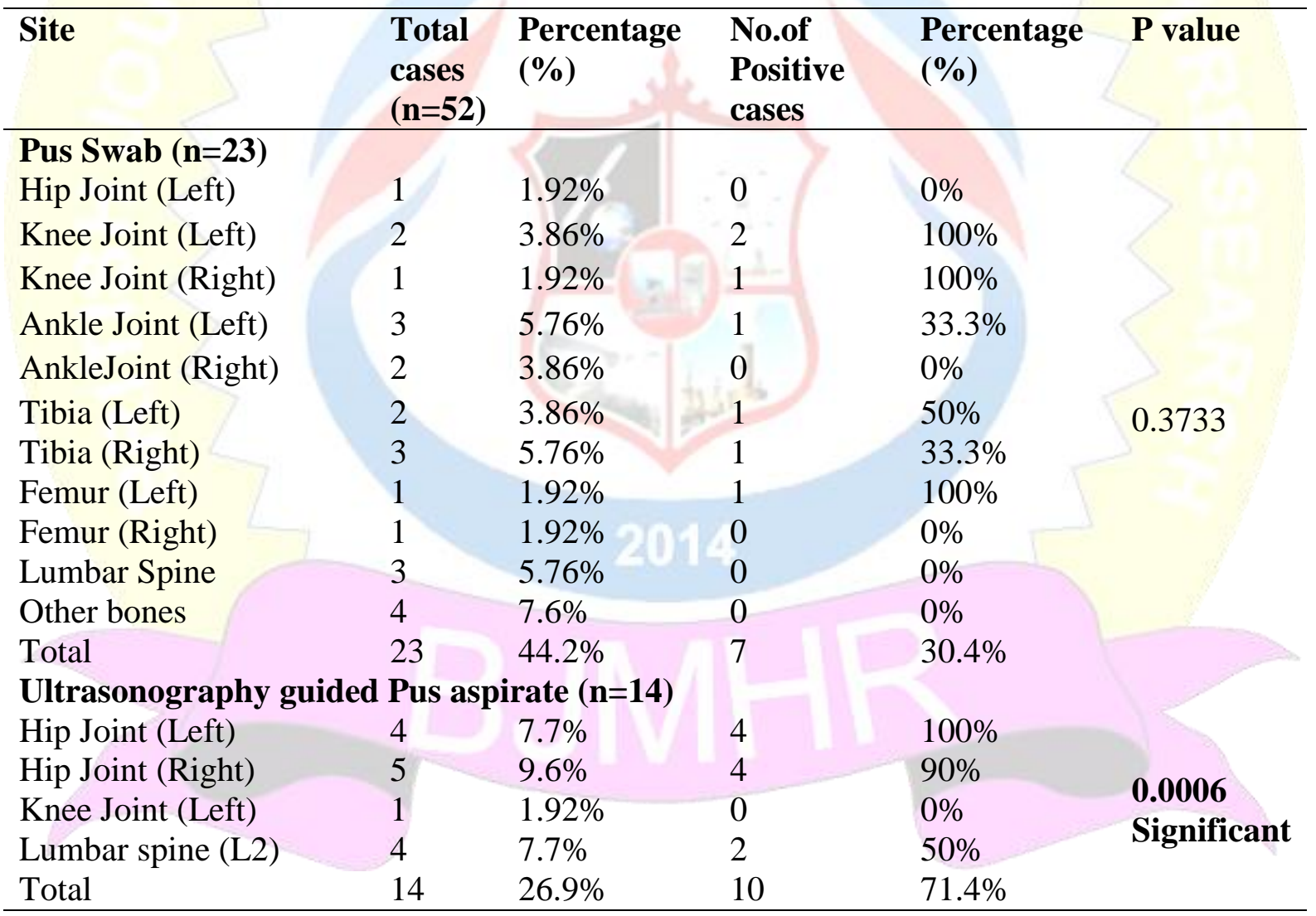

Table 2: Frequency of positivity of different tests for diagnosis of osteoarticular tuberculosis in various samples

\begin{tabular}{lllll}
\hline Sample & $\begin{array}{l}\text { No. of } \\
\text { cases }\end{array}$ & $\begin{array}{l}\text { AFB smear } \\
\text { Positive }\end{array}$ & $\begin{array}{l}\text { Culture on } \\
\text { LJ Number }\end{array}$ & $\begin{array}{l}\text { PCR } \\
\text { Number (\%) }\end{array}$ \\
\hline Pus swab & 23 & 0 & 3 & $7(30.4 \%)$ \\
USG guided pus aspiration & 14 & 1 & 5 & $10(71.4 \%)$ \\
\hline
\end{tabular}


Analysis of PCR results among specimens that were positive and negative by conventional bacteriological methods showed that one pus specimen was positive on smear microscopy and this specimen was positive on PCR (100\%). Among the negative specimens by smear microscopy, $45.1 \%$ of specimens were positive on PCR. This observation was in corroboration with the studies of Kumar et $\mathrm{al}^{15}$ (96\% in smear positive specimens and $46.3 \%$ in smear negative specimens) and Negi SS et $\mathrm{al}^{\mathbf{2 0}}$ (100\% in smear positive specimens and $35 \%$ in smear negative specimens). Similarly, in the present study PCR had $28.2 \%$ positivity among culture negative specimens and $100 \%$ positivity in positive specimens. These findings compare favorably with Kumar et $\mathbf{a l}^{\mathbf{1 5}}$ and Negi SS et $\mathbf{a l}^{\mathbf{2 0}}$.

\section{CONCLUSION}

The findings in our study can act as a bridge between early diagnosis of musculoskeletal tuberculosis at community or primary health care centre and prevention and treatment of deformity at the earliest possible, as these health care centres lack facility for clinical specimen collection from deep joints and diagnosis by conventional methods is difficult. Although the sample size in our study was small, it still highlights the importance of taking up more studies on a larger scale in this spectrum to help correlate the facts, uncover newer epidemiological and clinically relevant knowledge and thereby define the exact impact of the disease in our society and novel ways to decimate the same.

\section{Financial support and sponsorship}

I hereby extend my sincere gratitude towards State Tuberculosis Society, Meghalaya, India under RNTCP, GOI for financial support for this study.

\section{REFERENCES}

1. Revised National TB Control Programme Annual Status Report 2015, Government of India, Central TB division, Directorate General of Health Services, MOHFW, New Delhi -108, March 2016.

2. Raviglione MC, O’Brien RJ. Tuberculosis. In: Fauci AS, Braunwald E, Kasper DL, Hauser SL, Longo DL, Jameson JL, Loscalzo J (eds.) Harrison's Principles of Internal Medicine vol 1.19th ed. New York: McGraw Hill Inc; 2015:1102-1122.

3. Laboratory services in tuberculosis control. Culture Part III. World health organization 1998. WHO /TB/98/258.

4. Yoon HJ, Song YG, Park IW, Choi JP, Chang KH, Kim JM. Clinical Manifestations and Diagnosis of Extrapulmonary Tuberculosis. Yonsei Med J. 2004; 45:453- 61.

5. Sharma SK, Mohan A. Extrapulmonary tuberculosis. Indian J Med Res. 2004;120:316-53. 
6. Jutte PC, Van Loenhout Rooyackers JH, Borgdorff MW, Van Horn JR. Increase of bone and joint tuberculosis in The Netherlands, J Bone Joint Surg. 2004; 86(6):901904.

7. Nasiri MJ, Varahram M, Shams M, Taghavi K, Farnia P, Velayati AA. Osteoarticular Tuberculosis in Iran, 2002 to 2011, Advances in Research 2014;9(4): 509-14.

8. Enache SD, Pleasea IE, Anusca D, Zaharia B, Pop OT. Osteoarticular tuberculosis-a ten years case review Rom. J Morphol Embryol. 2005; 46:67- 72.

9. Arathi N., Faiyaz Ahmad, Najmul Huda. Osteoarticular tuberculosis a three years retrospective study. Journal of Clinical and Diagnostic Research 2013;7(10): 21892192.

10. Sharma V, Anjum R, Choudhary V, Singh TP. Epidemiological pattern of Osteoarticular Tuberculosis in a Teaching hospital of Rural India, International Journal of Biomedical Research 2016; 7(5): 273-275.

11. Ruiz G, Rodriguez GJ, Guerri ML, Gonzalez A. Osteoarticular tuberculosis in a general hospital during the last decade. Clin Microbiol Infect. 2003; 9:919-923.

12. Sandher DS, Al-Jibury M, Paton RW, Ormerod LP. Bone and joint tuberculosis: cases in Blackburn between 1988 and 2005. J Bone Joint Surg. 2007; 89: 1379-1381.

13. Prakash J. Diagnosis of Osteoarticular - Solution is in Peripheral Blood. J Microb Biochem Technol. 2016; 8:107-109

14. Ganavalli S. Ajantha, Praveen C. Shetty, Raghavendra D. Kulkarni, Umesh Biradar PCR as a diagnostic tool for extra-pulmonary tuberculosis. Journal of Clinical and Diagnostic Research 2013 ;7(6): 1012-1015.

15. Kumar M, Kumar R, Srivastava AK, Nag VL, Maurya AK, Dhole TN et al. Sensitivity of PCR IS6110 in relation to culture and staining in Pott's disease. Indian Journal of Neurosurgery 2013;2(1): 46-52.

16. Apurba SS, Sandhya BK. Comparison of conventional methods, BacT/ALERT and NESTED PCR for the diagnosis of tuberculous arthritis. International Journal of Basic Medical Science 2012;3(3): 81-86.

17. Wang G, Dong W, Zhao L, Yu X, Chen S, Fu Y, Qin S, Huang H. Evaluation of Lowenstein-Jensen Medium Culture, MGIT 960 Culture and Different Specimen Types in Diagnosis of Bone and Joint Tuberculosis. Journal of Pharmacy and Pharmacology 2015 3:128-132.

18. Muangchan C, Nilganuwong S. The study of clinical manifestation of osteoarticular tuberculosis in Siriraj Hospital, Thailand. J Med Assoc Thai. 2009; 92:101-09. 
19. Pandey V, Chawla K, Acharya K, Rao S. The role of polymerase chain reaction in the management of osteoarticular tuberculosis. Int Orthop. 2009;33(3):801-5.

20. Negi SS, Gupta S, Khare S, Lal S. Comparison of various microbiological tests including polymerase chain reaction for the diagnosis of osteoarticular tuberculosis. Indian J Med Microbiol. 2005; 23:245-248.

21. Maurya AK, Kant S, Nag VL, Kushwaha R, Dhole TN. Detection of 123 bp fragment of insertion element IS6110 Mycobacterium tuberculosis for diagnosis of extrapulmonary tuberculosis. Indian J Med Microbiol. 2012;30:182-6.

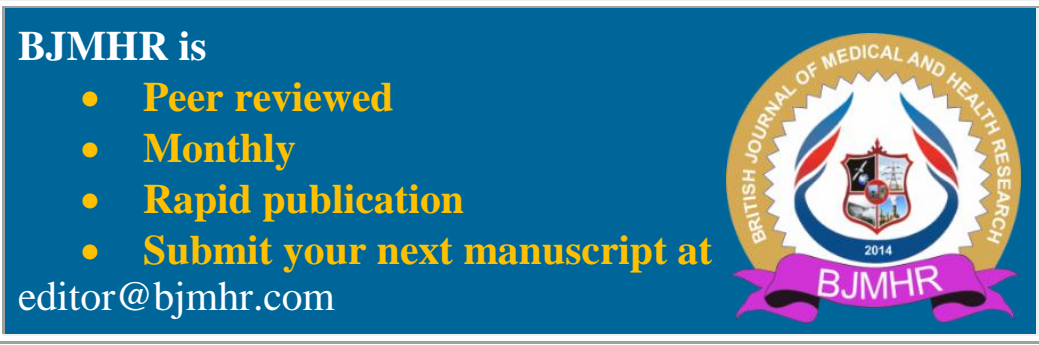

